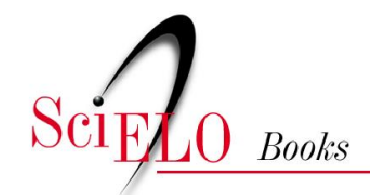

\title{
La investigación en educación
}

\author{
Maria Josefa Mosteiro García \\ Ana Maria Porto Castro
}

\section{SciELO Books / SciELO Livros / SciELO Libros}

GARCÍA, M. J. M., and CASTRO, A. M. P. La investigación en educación. In: MORORÓ, L. P., COUTO, M. E. S., and ASSIS, R. A. M., orgs. Notas teórico-metodológicas de pesquisas em educação: concepções e trajetórias [online]. Ilhéus, BA: EDITUS, 2017, pp. 13-40. ISBN: 978-857455-493-8. Available from: doi: 10.7476/9788574554938.001. Also available in ePUB from: http://books.scielo.org/id/yjxdq/epub/mororo-9788574554938.epub.

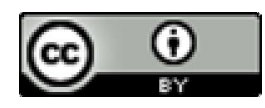

All the contents of this work, except where otherwise noted, is licensed under a Creative Commons Attribution $\underline{4.0 \text { International license. }}$

Todo o conteúdo deste trabalho, exceto quando houver ressalva, é publicado sob a licença Creative Commons Atribição 4.0.

Todo el contenido de esta obra, excepto donde se indique lo contrario, está bajo licencia de la licencia Creative Commons Reconocimento 4.0. 


\title{
stom \\ LA INVESTIGACIÓN EN EDUCACIÓN
}

\author{
Maria Josefa Mosteiro García ${ }^{1}$ \\ Ana Maria Porto Castro ${ }^{2}$
}

\section{La investigación en educación: origen y evolución}

La investigación educativa es una disciplina relativamente reciente. Su origen está marcado por la aparición de la pedagogía experimental en el siglo XIX, siglo que se caracteriza por el surgimiento de corrientes filosóficas como el positivismo de Comte (1789-1857), el pragmatismo del psicólogo norteamericano James, el sociologismo de Durkheim y el Empirismo de Dewey quien aplica la corriente pragmatista y establece en 1910 las etapas o fases básicas del pensamiento científico.

Otros acontecimientos especialmente significativos que podemos considerar como precedentes hacen alusión a los estudios de Weber (1795-1878), Ebbinghaus (1850-1878), Fechner (18011877), Wundt (1832-1920), Stanley Hall o Meumann, al igual que las aportaciones realizadas en el campo de la matemática por De Moivre, Gauss y Laplace y por Quetelet (1796-1874) precursor de la aplicación de la estadística a las Ciencias Sociales.

1 Professora na Universidad de Santiago de Compostela - Espanha.

E-mail: pepa.mosteiro@usc.es

2 Professora na. Universidad de Santiago de Compostela - Espanha.

E-mail: anamaria.porto@usc.es 
Podemos decir que es en los primeros ańos del siglo XX cuando el método experimental se constituye en el núcleo central de la investigación en educación. Este periodo inicial abarca aproximadamente el primer cuarto del siglo y representa un periodo de sistematización de la experimentación pedagógica, gracias a las aportaciones realizadas por Binet en Francia, iniciador del estudio científico del niño y su educación e introductor de las pruebas objetivas y los test y a los estudios de Galton en Inglaterra en torno a las diferencias individuales, los tests, la aplicación de la curva normal al estudio de los problemas psicológicos y el empleo de los métodos estadísticos.

Entre otras figuras pioneras cabe destacar en Alemania a Meumann que en el año 1900 acuña el término de pedagogía experimental y a Lay para quien la pedagogía experimental se define por la utilización del método de observación, la experimentación y la estadística. También cabe podemos citar a Cláparede, autor de la única obra hasta el año 1935 sobre métodos de investigación titulada Psychologie de L enfant et pédagogie expérimentale y a Thorndike, considerado como el representante más característico de la orientación científica en educación (LANDSHEERE, 1991).

Entre 1900 y 1930 destacan las contribuciones de los ingleses Pearson y Yule que permitirán el desarrollo de los principios de la correlación y la teoría de la regresión, así como las aportaciones de Fisher sobre los diseños, el análisis de varianza y covarianza, el análisis discriminante, etc.

Después de los años 30 la metodología científica va progresivamente madurando. En estos momentos destacan las contribuciones de autores como McCall quien, según Bartolomé (1987), puede ser considerado el sistematizador del método experimental en el ámbito de la educación. Aparecen trabajos sobre las propiedades matemáticas del azar con Eden y Yates (1931), Tedin (1931) y Barlett (1935) y se desarrollan las técnicas experimentales con los estudios de Campbell y Stanley.

Es a partir de la década de los cuarenta cuando la Pedagogía Experimental alcanza su madurez, coincidiendo con el importante 
desarrollo de la estadística, la sistematización de la teoría clásica de la medida con Gullicksen (1950) y con la aplicación de los principios del diseńo experimental gracias a las aportaciones de Linquist (1953).

En los años 60 destacan, sobre todo, los trabajos de Skinner y la corriente de análisis experimental del comportamiento, las aportaciones de Campbell (1963), de Cronbach (1974) y los primeros avances de la informática. El rechazo al reduccionismo positivista y al enfoque cuantitativo, predominante hasta entonces, abrirá un debate que dará lugar a un nuevo concepto de la investigación en educación, al enfrentarse una visión puramente numérica con una postura más cualitativa (PORTO, 1995, p. 27).

Por tanto, podemos decir que los cimientos de la investigación educativa, al menos en el ámbito anglosajón, tienen su base en la psicología educativa, la medida y evaluación y los métodos de investigación en educación (BARTOLOMÉ, 1987).

Paulatinamente, la investigación educativa ha seguido su desarrollo tras el agudo debate epistemológico acerca de la naturaleza de la ciencia; ha dado paso a la aparición de una nueva teoría de la medida; ha incorporado el uso de los ordenadores; ha planteado el pluralismo metodológico y, ha avanzado hacia una investigación más aplicada.

Al mismo tiempo, la expresión Pedagogía Experimental ha dado paso, tal y como indica Sandín (2003, p. 11) a otras denominaciones como "metodología de la investigación educativa", "fundamentos metodológicos de la investigación educativa", "investigación educativa", siendo este término el de uso más extendido en los últimos años.

Pero ¿Qué se entiende por investigación educativa? Para Cajide (1993) la investigación educativa se concibe dentro de las ciencias de la educación como una disciplina transversal que proporciona las bases metodológicas, con la finalidad de generar conocimientos específicos.

Según Escudero (1996), la investigación educativa es una disciplina que sustituye en terminología a la Pedagogía Experimental, que, a su vez, se separó a principios del siglo XX de la Psicología Experimental, con la que había realizado en común un largo recorrido. 
Autores como Sabariego y Bisquerra (2004, p. 37) nos hablan de la "investigación educativa como un conjunto sistemático de conocimientos acerca de la metodología científica aplicada a la investigación de carácter empírico sobre los diferentes aspectos relativos a la educación".

Por nuestra parte, entendemos la investigación educativa, siguiendo a Hernández Pina (1995, p. 3) como "El estudio de los métodos, procedimientos y las técnicas utilizadas para obtener un conocimiento, una explicación y una comprensión científicas de los fenómenos educativos, así como también para solucionar los problemas educativos y sociales". De acuerdo con esta propuesta, y tal como explica la propia autora, esta definición hace referencia al fenómeno o campo de estudio, la educación; los métodos, procedimientos y técnicas adecuadas, es decir, los métodos y metodología; y el propósito que se persigue, la creación y acumulación de conocimiento o la resolución de problemas.

\section{Perspectivas epistemológicas de la investigación en educación}

En los últimos años se han adoptado diferentes modos de acercamiento al estudio de la realidad educativa, fruto de los diferentes métodos, procedimientos y técnicas utilizadas para comprender los fenómenos educativos (HERNÁNDEZ PINA, 1995). Esto es, se han adoptado diferentes paradigmas que nos ofrecen diferentes maneras de "hacer" investigación y que se caracterizan según Lincoln y Guba (1985) por las respuestas que se ofrecen a tres cuestiones básicas relacionadas con la realidad que se desea estudiar, vinculadas con tres dimensiones:

- Ontológica (¿Cuál es la naturaleza de mi objeto de estudio?). Esta dimensión se refiere a la naturaleza de los fenómenos sociales.

- Epistemológica (¿Cuál es la relación entre el investigador/a y el 
objeto observado?). Esta dimensión alude al modo de relación entre quien investiga y dicha realidad.

- Metodológica (¿Cómo debería proceder el investigador/a?). Dimensión que hace referencia al modo en que debemos obtener conocimiento de dicha realidad.

En general se identifican tres paradigmas como marcos de referencia: el paradigma positivista, el paradigma interpretativo y el paradigma sociocrítico. Previamente al acercamiento de los aspectos claves de cada uno de ellos, es necesario detenernos en lo que entendemos por paradigma.

Etimológicamente, el vocablo paradigma procede del griego "paradeigma” y del latin "paradigma” que significa ejemplo, modelo. Siguiendo a Kuhn (1962, p. 271), "un paradigma es lo que comparten los miembros de una comunidad científica y, a la inversa una comunidad científica consiste en unas personas que comparten un paradigma”.

A partir de la definición de Kuhn (1962), otros/as muchos autores/as contribuyeron a difundir una visión de los distintos enfoques en investigación educativa basándose en este concepto Kuhniano. Entre las definiciones que mejor ilustran el sentido del término paradigma en el ámbito de la investigación educativa destacamos la siguiente: "Un punto de vista o modo de ver, analizar e interpretar los procesos educativos que tienen los miembros de una comunidad científica y que se caracteriza por el hecho de que tanto científicos como prácticos comparten un conjunto de valores, postulados, fines, normas, lenguajes, creencias y formas de percibir y comprender los procesos educacionales “ (DE MIGUEL, 1988, p. 66).

Por tanto, podemos decir que un paradigma nos muestra una determinada manera de concebir e interpretar la realidad, una visión del mundo compartida por un grupo de personas y que tiene un carácter normativo con relación a los métodos y técnicas de investigación a utilizar. 
El paradigma positivista, también denominado cuantitativo, empírico-analítico, es el paradigma dominante en algunas comunidades científicas, vinculándose a las ideas positivistas y empiristas de grandes teóricos del siglo XIX y principios del XX, como Comte (1798-1857), Mill (1806-1873), Durkheim (1858-1917) y Popper (1902).

Con esos supuestos teóricos de partida, el paradigma positivista incorpora el método hipotético-deductivo como procedimiento ampliamente aceptado en la investigación de las ciencias naturales y, posteriormente, en las ciencias sociales, cuya principal característica es la verificación rigurosa de proposiciones generales (hipótesis) a través de la observación empírica y el experimento en muestras de amplio alcance y desde una aproximación cuantitativa, con el fin de desarrollar algunas leyes a modo de explicación de los procesos educativos.

El propósito de la investigación se encamina a la explicación, el control, la comprobación y la predicción de los fenómenos educativos y actúa sobre el marco natural y externo al propio investigador, a quien lo que realmente le interesa es el producto final, esto es, el conocimiento observable, objetivo y cuantificable, con posibilidades de generalización.

Por su parte, el paradigma interpretativo, también denominado cualitativo, fenomenológico, naturalista, humanista o etnográfico engloba las corrientes humanísticas-interpretativas que centran su interés en el estudio de los significados de las acciones humanas y de la vida social.

Los orígenes de este paradigma se sitúan en Dilthey (18331911), Rickert (1863-1936), Schutz (1899-1959), Weber (18641920) y la fenomenología, el interaccionismo simbólico o la etnometodología.

Esta perspectiva sustituye las nociones científicas de explicación, predicción y control, propias del paradigma positivista, por la comprensión, el significado y la acción, penetrando en el mundo personal de los sujetos, en cómo interpretan las situaciones, en lo 
que significan para ellos y en qué intenciones tienen. Busca la objetividad en los significados, utilizando como criterio de evidencia el acuerdo intersubjetivo en el contexto educativo.

Este paradigma se concibe como una alternativa al positivista y se centra en la comprensión e interpretación de la realidad educativa desde los significados que las personas implicadas en los contextos educativos le atribuyen, estudiando sus creencias, intenciones, motivaciones no observables directamente, ni susceptibles de experimentación (LATORRE et al, 2003).

Finalmente, el paradigma sociocritico, pretende superar el reduccionismo del positivismo y el conservadurismo del paradigma interpretativo e introduce la ideología de forma explícita y la autorreflexión crítica en los procesos del conocimiento. Sus principios ideológicos tienen como finalidad transformar la estructura de las relaciones sociales, apoyándose en la Escuela de Franckfurt (Horkheimer, Adorno), en el neomarxismo (Apple, Giroux) o en la teoría crítica social de Habermas. Su objetivo es el análisis de las transformaciones sociales y dar respuesta a determinados problemas generados por éstas. Cuestiona la supuesta neutralidad de la ciencia y de la investigación, atribuyéndole un carácter emancipatorio y transformador de las organizaciones y procesos educativos. Es el propio grupo el que asume la responsabilidad de la investigación y el que propicia la reflexión y la crítica de los intereses, interrelaciones y prácticas educativas.

Algunos de los principios en los que se basa son: conocer y comprender la realidad como praxis; unir teoría y práctica, es decir, conocimiento, acción y valores; orientar el conocimiento a emancipar y liberar al hombre; implicar al docente a partir de la autorreflexión.

A modo de síntesis en la tabla 1 presentamos las principales características de los diferentes paradigmas. 
TABLA 1 - Características de los paradigmas de investigación

\begin{tabular}{|c|c|c|c|}
\hline \multirow{2}{*}{ Dimensión } & \multicolumn{3}{|l|}{ Paradigma } \\
\hline & Positivista & Interpretativo & Sociocrítico \\
\hline Fundamentos & $\begin{array}{l}\text { Positivismo lógico } \\
\text { Empirismo }\end{array}$ & $\begin{array}{l}\text { Fenomenología } \\
\text { Teoría } \\
\text { Interpretativa } \\
\end{array}$ & Teoría crítica \\
\hline $\begin{array}{l}\text { Naturaleza de la } \\
\text { realidad }\end{array}$ & $\begin{array}{l}\text { Objetiva, estática, } \\
\text { única,dada, } \\
\text { fragmentable, } \\
\text { convergente }\end{array}$ & $\begin{array}{l}\text { Dinámica, } \\
\text { múltiple, holística, } \\
\text { construida, } \\
\text { divergente }\end{array}$ & $\begin{array}{l}\text { Compartida, } \\
\text { histórica, } \\
\text { construida, } \\
\text { dinámica, } \\
\text { divergente }\end{array}$ \\
\hline $\begin{array}{l}\text { Finalidad de la } \\
\text { investigación }\end{array}$ & $\begin{array}{l}\text { Explicar, predecir, } \\
\text { controlar los } \\
\text { fenómenos, } \\
\text { verificar teorías. } \\
\text { Leyes para } \\
\text { regular los } \\
\text { fenómenos }\end{array}$ & $\begin{array}{l}\text { Comprobar } \\
\text { e interpretar } \\
\text { la realidad, los } \\
\text { significados de } \\
\text { las personas, } \\
\text { percepciones, } \\
\text { intenciones, } \\
\text { acciones }\end{array}$ & $\begin{array}{l}\text { Identificar } \\
\text { potencial } \\
\text { de cambio, } \\
\text { emancipar } \\
\text { sujetos. } \\
\text { Analizar la } \\
\text { realidad }\end{array}$ \\
\hline $\begin{array}{l}\text { Relación sujeto/ } \\
\text { objeto }\end{array}$ & $\begin{array}{l}\text { Independencia. } \\
\text { Neutralidad. } \\
\text { Sujeto/objeto } \\
\text { no se afectan. } \\
\text { Investigador } \\
\text { externo. Sujeto } \\
\text { como "objeto" } \\
\text { de investigación }\end{array}$ & $\begin{array}{l}\text { Dependencia. } \\
\text { Se afectan. } \\
\text { Implicación } \\
\text { investigador. } \\
\text { Interrelación }\end{array}$ & $\begin{array}{l}\text { Relación } \\
\text { influida por el } \\
\text { compromiso. } \\
\text { El investigador es } \\
\text { un sujeto más }\end{array}$ \\
\hline Valores & $\begin{array}{l}\text { Neutros. Investigador } \\
\text { libre de valores. Método } \\
\text { garantía de objetividad }\end{array}$ & $\begin{array}{l}\text { Explícitos. } \\
\text { Influyen en la } \\
\text { investigación }\end{array}$ & $\begin{array}{l}\text { Compartidos. } \\
\text { Ideología } \\
\text { compartida }\end{array}$ \\
\hline Teoría/práctica & $\begin{array}{l}\text { Disociadas, } \\
\text { constituyen } \\
\text { entidades distintas. } \\
\text { La teoría, norma para la } \\
\text { practica }\end{array}$ & $\begin{array}{l}\text { Relacionadas. } \\
\text { Retroalimentación } \\
\text { mutua. }\end{array}$ & $\begin{array}{l}\text { Indisociables. } \\
\text { Relación } \\
\text { dialéctica. La } \\
\text { práctica es teoría } \\
\text { en acción }\end{array}$ \\
\hline $\begin{array}{l}\text { Técnicas } \\
\text { Instrumentos } \\
\text { Estrategias }\end{array}$ & $\begin{array}{l}\text { Cuantitativos. Medición } \\
\text { de tests, cuestionarios, } \\
\text { observación sistemática. } \\
\text { Experimentación }\end{array}$ & $\begin{array}{l}\text { Cualitativos, des- } \\
\text { criptivos. } \\
\text { Invetigador pricipal } \\
\text { instrumento. } \\
\text { Perspectiva partici- } \\
\text { pantes }\end{array}$ & $\begin{array}{l}\text { Estudio de } \\
\text { casos. Técnicas } \\
\text { dialécticas }\end{array}$ \\
\hline Análisis de datos & $\begin{array}{l}\text { Cuantitativo: estadística } \\
\text { descriptiva e inferencial }\end{array}$ & $\begin{array}{l}\text { Cualitativo: } \\
\text { Inducción } \\
\text { analítica, } \\
\text { triangulación }\end{array}$ & $\begin{array}{l}\text { Intersubjetivo. } \\
\text { Dialéctico }\end{array}$ \\
\hline
\end{tabular}

Fuente: Latorre et al. (1996, p. 44) 
En suma, podemos decir que, en los últimos años, la investigación en el ámbito educativo se caracteriza por un pluralismo metodológico que permite la coexistencia de diversos enfoques (BARTOLOMÉ, 1992) el positivista o cuantitativo y el interpretativo y el crítico o sociocrítico, ambos integrados en lo que se suele denominar investigación cualitativa.

Las diferencias y el debate entre las distintas perspectivas se puede plantear tanto a nivel epistemológico como metodológico y técnico. En el plano epistemológico, el paradigma cuantitativo y cualitativo parten de supuestos filosóficos diferentes; desde el punto de vista metodológico, el problema fundamental es alcanzar un rigor científico; con respecto a las técnicas, son las características y exigencias de la propia investigación las que determinarán el uso de las mismas (PÉREZ SERRANO, 1994).

Desde una perspectiva histórica, la visión cuantitativa o positivista ha sido predominante en la investigación de las ciencias naturales y, también, en las ciencias sociales, y ha centrado sus esfuerzos en la verificación de hipótesis previamente establecidas como proposiciones cuantitativas, fácilmente transformables en términos matemáticos capaces de expresar relaciones funcionales. Pero, como resultado de las agudas críticas elevadas hacia la cuantificación, ha surgido una contrapartida a ésta, la investigación cualitativa que, con una amplia tradición en campos como la sociología y antropología, se ha convertido en una de las vías para acceder al conocimiento científico (BARTOLOMÉ, 1992). Al contrario que la investigación cuantitativa, centrada fundamentalmente en la medida y análisis de relaciones entre variables, la investigación cualitativa supone el estudio de un fenómeno en su marco natural, intentando darle sentido e interpretarlo en términos del significado que éste tiene para los propios actores. Puede ser considerada como un proceso activo, sistemático y riguroso de investigación, en el que se toman decisiones acerca de aquello sobre lo que se investiga mientras se está en el propio campo o ámbito objeto de estudio.

En concreto, de acuerdo con Denzin y Lincoln (1994) las diferencias entre ambos tipos de investigación, se refieren a: 
- Para los/as investigadores/as cuantitativos es posible estudiar, captar y comprender la realidad, mientras que los/as investigadores/ as cualitativos solo pueden aproximarse a esa realidad.

- Los investigadores/as cualitativos rechazan el uso de los métodos cuantitativos para el estudio de la sociedad.

- Ambos tipos de investigación se interesan por captar el punto de vista individual, pero en la cuantitativa es más difícil lograrlo por los materiales que utiliza y las inferencias que efectúa. La investigación cualitativa, a través de la entrevista y la observación, puede obtener una perspectiva del sujeto.

- La investigación cuantitativa es nomotética, basada en probabilidades derivadas del estudio de un gran número de casos seleccionados al azar. La cualitativa confronta las necesidades de la vida diaria.

- La investigación cuantitativa está menos interesada en el estudio de los detalles, la investigación cualitativa se preocupa por alcanzar una descripción minuciosa del mundo social.

De acuerdo con Guba y Lincoln (1994) las objeciones a la investigación cuantitativa se agrupan en dos grandes bloques, uno de ellos relacionado con las críticas internas, y el otro con las externas. En el primer bloque cabe señalar como principales objeciones la exclusión de significado; un contexto abierto; la disyunción de grandes teorías con contextos locales; la inaplicabilidad de los datos generales a casos individuales y la exclusión de la dimensión de descubrimiento en la investigación. Las críticas externas se refieren principalmente a la indeterminación de la teoría; al peso o valor de los hechos y a la naturaleza interactiva investigador-investigado.

Podemos decir también que la investigación cualitativa cuenta, por su parte, con algunas limitaciones derivadas, fundamentalmente, de la subjetividad que implican estos estudios, por la amplitud del objetivo y la complejidad de la situación a investigar.

Así pues podemos decir que tanto la investigación cuantitativa como la cualitativa presentan rasgos, características y limitaciones inherentes a su propia naturaleza. Los diversos paradigmas nos presentan diferentes modos de aproximarnos a la realidad educativa y 
ha sido precisamente esta casuística la que ha dado lugar a un debate paradigmático en torno a tres posturas (SABARIEGO, 2004, p. 77).

- Incompatibilidad entre paradigmas que compiten de manera irreconciliable en la investigación.

- Unidad epistemológica de la ciencia, en la que no se acepta la existencia de diversos paradigmas.

- Complementariedad entre paradigmas que, aunque de base ontológica y epistemológica distinta, se apoyan y complementan en el proceso de investigación.

No obstante, numerosos autores defienden la posibilidad de una complementariedad o conjunción de diversos procedimientos. Así, Bartolomé (1992, p. 15-16) indica que "tenemos que reconocer un pluralismo de enfoque en la investigación, el cual es consecuencia de la finalidad de la investigación, de los niveles de identificación entre investigador, investigado e informantes, de los criterios de cientificidad y de las tradiciones disciplinares, ideologías o grupos científicos que la sustenta”.

En este sentido, hay que precisar que existen diferentes posturas. En ocasiones, la investigación cuantitativa y la cualitativa se presentan en términos opuestos y de dicotomía. En otros casos se señala la posibilidad de utilizar los distintos enfoques, dado que los procesos educativos pueden entenderse bajo una amplia dimensionalidad. Como dice Husen (1988, p. 589) "los enfoques metodológicos ya los designemos o no como paradigmas, no se hallan forzosamente en conflicto entre sî".

En esta misma línea se manifiesta Anguera (1986) defendiendo, para obtener una mayor riqueza informativa, la complementación entre datos cuantitativos y cualitativos, por ejemplo a través del uso de modelos causales basados en observaciones.

En síntesis es preciso reconocer que la realidad educativa está integrada por elementos diversos y, por ello, puede ser abordada desde diferentes procedimientos de distinto carácter. Así, podemos concluir que en el ámbito de la investigación educativa 
hoy en día está aceptada una actitud integradora, en las que las distintas aproximaciones se complementan porque, como afirma Sechrest y Sidani (1995, p. 77) "la buena ciencia se caracteriza por el pluralismo metodológico”. Esto creemos que es sumamente importante porque la metodología científica nunca fue una realidad cerrada, sino que, por el contrario, siempre ha sido una realidad abierta tal y como refleja la historia de la ciencia.

\section{Métodos de investigación en educación}

Una de las cuestiones más controvertidas en la construcción del conocimiento, en nuestro caso el conocimiento de la educación, es la del método o de la metodología de investigación a utilizar para su elaboración.

En su origen el concepto de método venía a significar el camino que se recorre para alcanzar un objetivo o un fin establecido previamente, o el procedimiento que se realiza según un conjunto de normas. En este sentido, método es equiparable a orden, dirección, guía reglamentación, etc. y se opone, por tanto, a azar, desorden, etc. (FERRATER, 1979; ARNAL, DEL RINCÓN y LATORRE, 1992).

El método es definido por Bunge (1979, p. 25) como "el procedimiento para tratar un conjunto de problemas". En esta misma línea Van Dalen y Meyer (1981, p. 42) entienden el método como "una poderosa y útil antorcha que nos puede alumbrar el camino en la búsqueda de nuevos conocimientos".

El Diccionario de la lengua espańola define de manera general el método como el "modo de obrar o proceder, hábito o costumbre que cada uno tiene y observa” y más específicamente, como "procedimiento que se sigue en las ciencias para hallar la verdad y enseñarla". Para Colás (1992, p. 61) "el método son las distintas formas en las que puede o debe plasmarse en cada caso, la noción general de lo que veníamos llamando método científico". 
Como podemos comprobar, en todas estas definiciones de método se encuentran los mismos presupuestos: orden, sistema, camino, fin, propósito, procedimiento, todo lo contrario a desorden, azar, suerte, es decir, comparten unos elementos que se constituyen en esenciales a la hora de entender el concepto de método. Estos elementos son los siguientes:

- Debe existir un fin o propósito al que se pretende llegar y, por consiguiente, conocido. En este sentido, se puede decir que es intencional.

- Debe mantenerse un orden. Este es quizá el elemento mayoritariamente utilizado en las definiciones y, de este modo, se podría entender que sin ese orden no es posible el método o, si se prefiere, no es posible su aplicación.

- Es complejo, ya que está compuesto de múltiples y diferentes elementos que producen diversos órdenes.

Entendemos, por tanto, que uno de los elementos fundamentales del trabajo educativo es el método, porque con toda intervención se pretende alcanzar un determinado resultado. Así, los métodos de investigación están destinados, fundamentalmente, a aumentar y/o profundizar el conocimiento de la realidad, llevando a cabo nuevos descubrimientos, nuevas invenciones o reinterpretando la realidad ya conocida.

Cuando hablamos de los métodos de investigación es fundamental establecer las diferencias entre método, técnica y metodología. "El concepto de metodología significa el estudio del método, la lógica de la investigación, la lógica de los métodos; se refiere al modo de enfocar los problemas y de buscarle respuestas" (SABARIEGO, 2004, p. 79). El método, tal y como dijimos anteriormente, se refiere al camino para alcanzar los fines de la investigación y está definido por su carácter regular, explícito, repetible, racional, ordenado y objetivo para lograrlo. Finalmente, las técnicas se entienden como "los instrumentos, las estrategias y los análisis documentales empleados por los investigadores para la recogida de información” (Del Rincón et al., 1995, p. 35). 
De modo más concreto podemos decir que los distintos enfoques o perspectivas de investigación educativa (paradigmas) aportan una diversidad de metodologías y, a su vez, cada una de estas metodologías incluyen distintos métodos o tipos de investigación, con una variedad de técnicas particulares, de carácter más práctico y operativo, que permiten hacer efectivo su desarrollo (SABARIEGO, 2004).

Pero, además de la metodología abierta, el pluralismo metodológico implica también la existencia de diferentes métodos. Parece claro que, en función de los diferentes fines que se fijen y de la naturaleza del objeto a analizar, caben diferentes métodos. En esta línea se sitúa Kerlinger (1979, p.4) cuando nos dice "que no hay un único método científico como tal. Hay muchos métodos que los hombres de ciencia pueden utilizar y de hecho se emplean". Del mismo modo, Travers (1971, p. 16) indica que "se llega al conocimiento científico mediante una variedad de procedimientos y métodos".

Cada método presenta unas posibilidades y limitaciones, dependiendo su uso de la forma de investigar y de las metas que en la investigación se persigan. Además, el pluralismo metodológico nos ofrece la posibilidad de trascender muchos problemas que son inherentes al uso de métodos simples y reducidos.

Ahora bien, describir los distintos métodos de investigación en el ámbito educativo no es una tarea sencilla debido, principalmente, a la diversidad de criterios y categorías clasificatorias. Así, algunas clasificaciones parten de un único criterio mientras otras combinan diversas reglas, por ejemplo, la forma de recogida de datos, los objetivos del método, etc. (VAN DALEN y MEYER, 1981; DE LA ORDEN, 1989; COLÁS, 1992).

En esta línea, conscientes de las múltiples y variadas clasificaciones efectuadas hasta el momento, agrupamos los distintos métodos de investigación en educación en dos grandes bloques. Así, hablamos de métodos de investigación cuantitativos entre los que cabe incluir el método experimental, descriptivo y correlacional y de los métodos de investigación cualitativa, entre los que situamos la investigación etnográfica, la investigación-acción y el estudio de casos. 
En términos generales, los métodos de investigación cuantitativos se caracterizan fundamentalmente por el uso de datos numéricos que representan cantidades de aquello que se mide. El método experimental "intenta buscar los fenómenos que se derivan de la manipulación de ciertas condiciones" (TEJEDOR, 1994, p. 263). Un experimento es una modalidad de investigación en la que se manipula una o más variables independientes y en el que se minimizan la influencia de las variables extrañas, con la finalidad de estudiar la relación entre dos o más variables. La persona investigadora, en este caso, para someter a prueba la hipótesis de investigación recurre a la experiencia controlada, al experimento, ya que este aporta a la investigación control y rigor.

Pero, lo que realmente da relevancia a la investigación experimental son sus características, entre las que podemos destacar: el control, la aleatoriedad, la replicación, la flexibilidad, la eficacia, la potencialidad y la comparación.

Por su parte, el método descriptivo pretende describir un fenómeno analizando su estructura y explorando las asociaciones relativamente estables de las características que lo definen. En este sentido, es un método inductivo. Su objetivo fundamental es llegar a conocer las situaciones, costumbres y actitudes predominantes, mediante la descripción de actividades, objetos, procesos y personas utilizando, para ello, técnicas de recogida de datos como la observación o la encuesta. Según Arnal et al. (1992) dentro de la investigación descriptiva podemos englobar los siguientes tipos de estudios: estudios tipo encuesta, estudios de desarrollo y estudios observacionales.

Los métodos correlacionales son contemplados como un puente de unión entre los métodos experimentales y los descriptivos; pretenden descubrir las relaciones entre variables o bien probar las relaciones que intervienen en un fenómeno. Implican el cálculo de un coeficiente de correlación, como medida de la extensión en la que las variables varían conjuntamente. Básicamente se utilizan para efectuar exploraciones iniciales de las relaciones entre variables. 
En cuanto a los métodos cualitativos, en general, se caracterizan por la inmersión del investigador/a en el marco de investigación y por los esfuerzos que éste/a hace para descubrir el significado y el significante que el fenómeno social tiene para las personas.

La investigación etnográfica es un "tipo de investigación que se realiza en un ámbito relativamente pequeño, homogéneo en sus características poblacionales y limitado geográficamente, que emplea como técnica fundamental la observación y, en concreto, la observación participativa, y que tiene como objetivo describir, interpretar y explicar la cultura y la estructura social de la población a estudiar" (Porto, 1995, p. 106).

La investigación-acción tiene como objeto mejorar la educación mediante su cambio y lograr un aprendizaje a través de éste. Su finalidad no es la acumulación de conocimientos sobre la enseñanza o la comprensión de la realidad educativa, sino aportar información que guíe la toma de decisiones y los procesos de cambio para la mejora de la misma. Para ello utiliza un procedimiento de investigación en espiral (empleo de etapas o ciclos sucesivos: planificación/acción/observación/reflexión), una búsqueda rigurosa y sistemática del conocimiento de la educación y mejora de la práctica educativa basada en un proceso de autorreflexión de los agentes sobre sus propias acciones.

El estudio de casos es un estudio intensivo, longitudinal e ideográfico que representa una alternativa a los estudios de carácter nomotético. Sus rasgos característicos lo convierten en un método especialmente indicado en el ámbito educativo, en el que el investigador ha de enfrentarse al estudio de una situación no estructurada ni sistemática (PÉREZ JUSTE, 1994). En términos generales, podemos decir que este método implica un proceso de indagación caracterizado por el examen detallado, comprehensivo, sistemático y en profundidad del caso objeto de estudio. 


\section{$4 \mathrm{El}$ proceso de investigación en educación}

Para que la investigación educativa podamos considerarla como tal, debemos recurrir al método científico consistente en una secuencia de pasos aceptados y adoptados por la comunidad científica o como dice McMillan Y Schumacher (2005, p. 16) "en una secuencia orientativa que se va modulando en función del desarrollo de la misma”. Son varias las propuestas realizadas por diferentes autores en relación con las fases a seguir en el proceso de investigación. En líneas generales, todas las propuestas coinciden en los puntos básicos, aunque se pueden constatar la existencia de diferencias. En general, podemos diferenciar cinco grandes fases en el proceso de investigación que se concretan, a su vez, en una serie de etapas: clarificación e identificación del problema; planificación de la investigación; planificación de la recogida de datos; organización y procesamiento de los datos y difusión de los resultados. 
FIGURA 1 - Fases del proceso de investigación educativa

\section{PRIMERA FASE}

Clarificación e Identificación del Problema

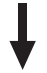

Delimitación del tema

Formulación del problema

Revisión de fuentes bibliográficas

SEGUNDA FASE

Planificación de la Investigación

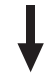

Formulación de objetivos e hipótesis

Definición y categorización de las variables

Elección del método y del diseño

Población y Muestra

\section{TERCERA FASE}

Planificación de la recogida de datos

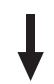

Selección del instrumento de recogida de datos

\section{CUARTA FASE}

Organización y Procesamiento de los datos

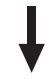

Análisis de los datos

Resultados y Conclusiones

\section{QUINTA FASE}

Difusión de los Resultados

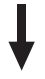

Informe de Investigación

Fuente: Elaboración propia 


\subsection{Primera Fase: Clarificación e identificación del problema}

Esta etapa abarca la identificación del problema y del conocimiento relacionado con el mismo a través de la revisión de fuentes bibliográficas con el fin de enmarcarlo teórica y empíricamente.

\subsubsection{Delimitación del tema}

El punto de partida de toda investigación científica es el interés en un tema o en un área temática amplia. Booth et al. (2001) definen el tema o la idea inicial de investigación como aquella cuestión o asunto que se va a estudiar, con interés suficiente para sustentar investigaciones que contribuyan a hacer progresar la comprensión del mismo. Existe uma gran variedad de fuentes que pueden generar ideas de investigación, por ejemplo, experiencias individuales, materiales escritos, materiales audiovisuales, conversaciones personales, observaciones de hechos, etc.

\subsubsection{Formulación del problema}

Una vez selecionado el tema, y para poder continuar con la investigación, hay que precisar la idea de investigación. Dicho processo se denomina identificación o planteamiento del problema. El problema de investigación es una pregunta para la que no se tiene respuesta. Puede surgir por una laguna en el conocimiento, por existencia de contradicciones em investigaciones anteriores o bien porque existe un vacío o laguna en los resultados de otras investigaciones. Un problema se caracteriza por ser resoluble, relevante, factible, claro y preciso.

\subsubsection{Revisión de Fuentes bibliográficas}

Tras delimitar el tema objeto de estudio y formular el problema de investigación, el paso siguiente consiste en realizar una revisión de la literatura sobre el tema. La revisión documental permite 
interpretar con mayor fundamento el significado de los resultados y evita descubrir cosas ya descubiertas por otros. Proporciona también la perspectiva o el marco de referencia conceptual e indicaciones y sugerencias para plantear el enfoque, el método o las técnicas de recogida de información (Latorre et al., 2003).

\subsection{Segunda Fase: Planificación de la Investigación}

Esta fase supone la formulación de los objetivos, las hipótesis, la definición y la categorización de las variables, así como la elección del método, el diseño y la población y la muestra.

4.2.1 Formulación de objetivos e hipótesis

Los objetivos de la investigación tienen la finalidad de señalar lo que se pretende y a lo que se aspira alcanzar en la investigación.

Después de haberse preguntado qué investigar y buscando la determinación del problema se deben formular las hipótesis de investigación. Las hipótesis científicas son, en general, suposiciones. Desde este punto de vista son enunciados teóricos supuestos, no verificados pero probables, referentes a variables o a la relación entre variables.

\subsubsection{Definición y categorización de las variables}

Una variable es una característica o atributo que puede tomar diferentes valores o modalidades.

Las variables presentan, tomadas separadamente, dos características fundamentales: ser características observables de algo y ser susceptibles de cambio o variación con relación al mismo o diferentes objetos.

\subsubsection{Elección del Método}

Una vez delimitado el problema de investigación, formuladas las hipótesis, y definidas las variables, la persona investigadora debe 
seleccionar el método que mejor se adecúe y responda a la pregunta de investigación. El método es el conjunto de procedimientos que permiten abordar un problema de investigación con el fin de lograr unos objetivos determinados, es decir, sirve de instrumento para alcanzar los fines de la investigación.

\subsubsection{Elección del Diseño}

Una vez selecionado el método más adecuado para la investigación hay que selecionar el diseño de la investigación y aplicarlo al contexto particular del estudio. El diseño es el plan o estrategia a seguir para obtener la información que se desea, para dar respuesta al problema planteado, cumplir los objetivos del estúdio y someter a prueba las hipótesis. Cada uno de los métodos se concreta en diseños con características propias. El diseño que se utilice debe ser apropiado a la investigación y el investigador/a debe siempre buscar el diseño más simple y económico que le ayude a responder a su problema de investigación.

\subsubsection{Población y Muestra}

La población se refiere al conjunto de todos los individuos en los que se desea estudiar un fenómeno. La muestra es el subconjunto de individuos extraídos de la población que se selecciona a través de alguna técnica de muestreo. Para que se puedan generalizar a la población los resultados obtenidos en la muestra, ésta ha de ser representativa y debe tener un tamańo suficiente para garantizar esa representatividad. El procedimiento para seleccionar la muestra de individuos sobre los que se van a recoger los datos se denomina muestreo. Podemos clasificar los principales procedimientos de muestreo en dos: muestro probabilístico, aquel que selecciona la muestra al azar de modo que todos los individuos de la población tienen las mismas probabilidades de entrar a formar parte de ella, y muestreo no probabilístico, aquel en el que la selección de los 
individuos de la muestra no depende de la probabilidad, sino que se ajusta a otros criterios relacionados con las características de la investigación o de quien la realiza.

\subsection{Tercera Fase: Planificación de la recogida de datos}

Una vez seleccionada la muestra, el siguiente paso es la planificación de la recogida de datos y la selección de las técnicas de obtención de la información más adecuadas al problema objeto de estudio.

\subsubsection{Selección del instrumento de recogida de datos}

"Los instrumentos son médios reales, con entidad propia, que las investigadoras e investigadores elaboran con el propósito de registrar información y o medir características de los sujetos" (SABARIEGO, 2004, p.150). Disponemos de una gran variedad de instrumentos tanto cuantitativos (cuestionarios, observación estructurada, escalas, registros...) como cualitativos (entrevista, observación participacion...) y en un mismo estudio podemos utilizar los dos tipos de instrumentos. La persona que investiga puede selecionar los instrumentos entre los ya existentes o bien elaborar los suyos propios. Al margen del tipo de instrumento que utilicemos, todos deben reunir dos requisitos esenciales, ser fiables y ser válidos.

\subsection{Cuarta Fase: Organización y procesamiento de los datos}

Esta fase abarca el análisis de los datos y la presentación de los resultados y conclusiones. 
El propósito fundamental de la fase de análisis de datos consiste en dar sentido a la información obtenida, tratándola y organizándola para poder explicar, predecir e interpretar el fenómeno objeto de estudio y dar respuesta al problema planteado, es decir, ayudar a dar sentido y a contrastar empíricamente las hipótesis de trabajo.

En función de la información obtenida podemos distinguir dos tipos de análisis, el cuantitativo y el cualitativo. En el primero se suelen aplicar métodos estadísticos para analizar datos numéricos, expresar las leyes generales que rigen los fenómenos educativos, decidir si las hipótesis se confirman o se rechazan y llegar a una generalización de los resultados. Para todo ello se utiliza la estadística descriptiva y la estadística inferencial.

Desde el enfoque cualitativo, el análisis se efectúa sobre datos de una naturaleza distinta; los datos cualitativos son textos procedentes de entrevistas o sesiones de observación y vienen expresados en un lenguaje natural.

A diferencia del análisis cuantitativo el cualitativo transcurre simultáneamente a la obtención de la información y consiste en un procedimiento abierto y flexible para la clasificación de los datos de acuerdo con unidades básicas de significado a fin de resumirlos y tabularlos.

\subsubsection{Resultados y Conclusiones}

Tras el análisis de la información recogida los resultados obtenidos deberán exponerse de la forma más completa y precisa en tablas, cuadros, diagramas o cualquier otro procedimiento gráfico generado por el análisis realizado. Junto a los resultados pueden incluirse las conclusiones de la investigación, en las que se recoge una interpretación no técnica de los resultados, es decir, en este apartado el investigador o investigadora explica el significado 
que tienen los resultados respeto de los objetivos y las hipótesis suscitadas e interpreta los fenómenos y procesos observados desde determinados marcos teóricos y las coincidencias o discrepancias respeto a estudios similares. También se pueden incluir en las conclusiones interrogantes o nuevas y futuras líneas de investigación.

\subsection{Quinta Fase: Difusión de los resultados}

El proceso de investigación concluye con la difusión de los resultados obtenidos a través de la redacción de un informe (normalmente escrito) en el que se transmite lo realizado y las conclusiones alcanzadas.

\subsubsection{Informe de investigación}

La redacción del informe reviste gran importancia en cualquier trabajo de investigación. En esta etapa el/a autor/a informa a la comunidade científica lo que ha hecho y como ha llevado a cabo su trabajo y, al mismo tempo, da la oportunidad a los miembros de la comunidad científica de criticar y debatir tanto los resultados como los procedimientos utilizados en su obtención, y también, la posibilidad de poder replicar empíricamente el trabajo presentado. Habitualmente en un informe de investigación se recogen los siguientes apartados: título, autor/a, filiación, resumen o abstract, la introducción, el método, los resultados, discusión y conclusiones, referencias bibliográficas, anexos y apéndices. 


\section{Referencias}

ANGUERA, Ma. T. La investigación cualitativa. Educar, 10, 23-10, 1986.

ARNAL, J.; DEL RINCÓN, D.; LATORRE, A. Investigación educativa. Metodologías de investigación educativa. Barcelona: Labor, 1992.

ARNAL, J.; RINCON, D.; LA TORRE, A. Investigación educativa. Fundamentos y metodologia. Barcelona: Labor, 1992.

BARTOLOMÉ PINA, M. Investigación cualitativa en educación, comprender o transformar. Revista de Investigación Educativa, 20 (2), 7-36, 1992.

BARTOLOMÉ PINA, M. La pedagogia experimental. En: SANVISENS, A. (Ed.) Introducción a la investigación pedagógica (2a ed.). Barcelona: Barcanova, 1984. p. 381-403.

BARTOLOMÉ PINA, M. Metodología qualitativa orientada cap al canvi i la presa de decisions. Temes universitaris bàsics, Psicopedagogia. Barcelona: Edicions de la Universitat Oberta de Catalunya, no 96, 1987.

BOOTH, W.; COLOMB, G.; WILIAMS, J.M. Como convertirse en un hábil investigador. Barcelona: Editorial Gedisa, 2001.

BUNGE, M. La investigación científica. Su estrategia y su filosofía. Barcelona: Ariel, 1979.

CAJIDE VAL, J. Proyecto Docente e Investigador de Pedagogía Experimental. Santiago de Compostela: Universidad de Santiago de Compostela, 1993.

COLÁS BRAVO, Ma . P. Los métodos de investigación en educación. En: COLÁS, Ma P.; BUENDÍA, L. (Eds.) Investigación Educativa. Sevilla: Alfar, 1992. p. 43-67. 
DE LA ORDEN, A. Investigación cuantitativa y medida en educación. Bordón, 41(2), 217-235, 1989.

DE MIGUEL, M. Paradigmas de la investigación educativa española. En: Dendaluce, I. (Ed.), Aspectos metodológicos de la investigación educativa Madrid: Narcea, 1988, p. 60-77.

DEL RINCÓN, D.; ARNAL, J.; LATORRE, A.; SANS, A. Técnicas de investigación en ciencias sociales. Madrid: Dykinson, 1995.

DENZIN, N.K.; LINCOLN, Y.S (Eds.) Handbook of qualitative research. London: Sage Publications, 1994.

ESCUDERO, T. Proyecto Docente e Investigador. Investigación evaluativa en el Instituto de Ciencias de la Educación. Zaragoza: Universidad de Zaragoza, 1996.

FERRATER, J. Diccionario de filosofia. Madrid: Alianza Editorial, 1979.

GUBA. E.G.; LINCOLN, Y.S. Competing paradigms in qualitative research. En: N. K. DENZIN, N.K.; LINCOLN, Y.S. (Eds.) Handbook of qualitative research. London: Sage Publication, 1994. p. 105-117.

HERNÁNDEZ PINA, F. Bases metodológicas de la investigación educativa. Barcelona: PPU, 1995.

HUSEN, T. Paradigmas de la investigación en educación: un informe del estado de la cuestión. En: Dendaluce, I. (Ed.) Aspectos metodológicos de la investigación educativa. Madrid: Narcea, 1988. p. 46-59.

KERLINGER, F. N. Investigación del comportamiento. Técnicas y metodologia (2a ed.). México: Interamericana, 1979.

KUHN, T.S. La estructura de las revoluciones científicas. México: Fondo de Cultura Económica, 1962.

LATORRE, A.; DEL RINCÓN, D.; ARNAL, J. Bases metodológicas de la investigación educativa. Barcelona: Hurtado Ediciones, 1996. 
LATORRE, A.; DEL RINCÓN, D.; ARNAL, J. Bases metodológicas de la investigación educativa. Barcelona: Ediciones Experiencia, 2003.

LINCOLN, Y.S. y GUBA, E.G. Naturalistic Inquiry. Beverly Hills: Sage, 1985.

MCMILLAN, J.H. y SCHUMACHER, S. Investigación educativa. Madrid: Pearson, 2005.

PERÉZ JUSTE, R. El estudio de casos. Diseño de caso único. En: GARCÍA HOZ, V. (Dir.) Problemas y métodos de investigación em educación personalizada. Madrid: Rialp, 1994. p. 475-509.

PÉREZ SERRANO, G. Investigación cualitativa: retos e interrogantes. I Métodos. II Técnicas y Análisis de Datos. Madrid: La Muralla, 1994.

PORTO, A. Proyecto Docente de Pedagogía Experimental. Santiago de Compostela: Universidad de Santiago de Compostela, 1995.

SABARIEGO, M. El proceso de investigación (Parte 2). En: BISQUERRA, R. (Coord.) Metodología de la Investigación Educativa. Madrid: La Muralla, 2004. p. 127-163.

SABARIEGO, M. La Investigación educativa: génesis, evolución y características. En: BISQUERRA, R. (Coord.) Metodología de la Investigación Educativa. Madrid: La Muralla, 2004. p. 51-87.

SABARIEGO, M. y BISQUERRA, R. Fundamentos metodológicos de la Investigación Educativa. En: BISQUERRA, R. (Coord.) Metodología de la Investigación Educativa. Madrid: La Muralla, 2004. p. 19-49.

SANDÍN, M.P. Investigación cualitativa en educación. Fundamentos y tradiciones. Madrid: McGraw-Hill, 2003.

SECHREST, L.; SIDANI, S. Quantitative na qualitative methods: Is there na alternative?. Evaluation na Program Planning, 18 (1), 77-87, 1995. 
TEJEDOR, F. J. La experimentacion como método de investigación. En: GARCÍA HOZ, V.(Dir.) Problemas y métodos de investigación en educación personalizada. Madrid: Rialp, 1994. p. 256-280.

TOJAR, J.C. Investigacion cualitativa: comprender y actuar. Madrid: La Muralla, 2006.

TRAVERS, R.M. Introducción a la investigación educacional. Buenos Aires: Paidós, 1971.

VAN DALEN, D. E.; MEYER, W. Jh. Manual de técnicas de investigación educacional. Buenos Aires: Paidós, 1981. 